\title{
Progressive Limb Weakness and Sensory Loss in a Young Woman
}

\author{
Hannah Glass $^{\S}$, B.A., Myriam Srour ${ }^{\S}$ B.Sc., Giovanna Pari ${ }^{\dagger}$, M.D., F.R.C.P.(C.), \\ George Karpati ${ }^{\dagger}$, M.D., F.R.C.P.(C.), and G. Jackson Snipes ${ }^{* \dagger}$, M.D., Ph.D.
}

\section{INTRODUCTION}

Patients with neuromuscular disease often present with a combination of symptoms that suggest a wide differential diagnosis. Traditionally, electrophysiologic studies and microscopy have aided the clinician in making a diagnosis. More recently, genetic testing for specific diseases has helped to ensure correct diagnosis. The following is a case that emphasizes the importance of combining clinical, electrophysiologic, microscopic and finally, genetic findings.

\section{THE CASE}

A 37-year-old woman presented with fluctuating symptoms of limb weakness and numbness. She first noticed left leg atrophy and weakness at the age of 33, with progression to the right leg one year later. The patient also described weakness in her right arm for the last two years and in the left arm for less than a year. During the last four years, she had numbness in both hands, the right starting before the left, with some fluctuation of symptoms. She currently wears orthoses for a foot drop, which was first noted 16 years ago. As a young child, she attained motor milestones at a normal age and later was able to participate in athletic activities, but had a tendency to trip over her feet. Past medical history reveals hypothyroidism and depression, for which she takes Levothyroxine and Paroxetine. One of the patient's three children has reduced muscle mass

\footnotetext{
* To whom correspondence should be addressed:Dr. G. Jackson Snipes, 3801 University Street, Montreal, Quebec, Canada, H3A 2B4. E-mail: jsnipes@mni.lan.mcgill.ca.

$\S$ Faculty of Medicine, McGill University.

$\dagger$ Neuromuscular Research Group, Department of Neurology and Neurosurgery, Montreal Neurological Institute, McGill University.
}

in both legs and possible weakness. No other relatives appear to be affected.

On clinical examination, the patient appeared wellbuilt and of stated age. Her respiratory, cardiovascular and abdominal examinations were within normal limits. The neurological examination provided significant findings. The cranial nerves were normal. The motor examination showed prominent atrophy in the lower extremities, as well as pes cavus (exaggeration of the arch of the foot) and hammer toes. Power testing revealed mild weakness in the distal upper extremities (intrinsic hand muscles $4 / 5$ bilaterally) and in the proximal lower extremities (quadriceps and hamstrings $4 / 5$ bilaterally), as well as significant weakness in the distal lower extremities (tibialis anterior 1/5 bilaterally). Tendon reflexes were normal except for the ankle jerks, which were absent bilaterally. The plantar response was inflexion. There was markedly impaired sense of vibration and joint position in the feet. There was a diminution of sensitivity to light touch in the legs to the inguinal area bilaterally. Superficial pain sensation was absent in a stocking distribution over the legs up to the level of the knees and over the hands up to the wrists bilaterally. The cerebellar examination was within normal limits.

Laboratory investigations included a normal immunological profile (erythrocyte sedimentation rate, serum protein electrophoresis, complement, antinuclear antibody, and rheumatoid factor), as well as normal thyroid function tests and creatine kinase activity. Cerebrospinal fluid studies, including protein level, were normal.

Electrophysiologic studies were used to help differentiate whether the patient's deficits were myogenic or neurogenic. Electromyography (EMG) of 


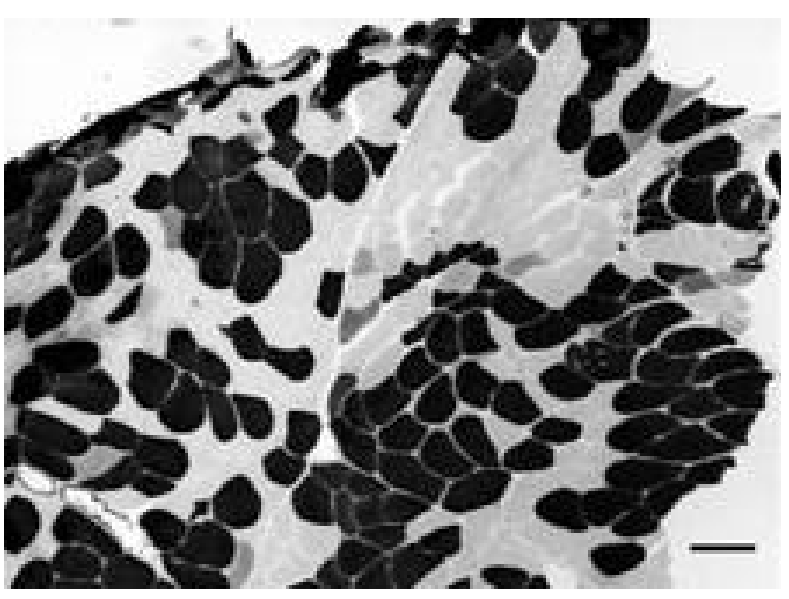

Figure 1. Cross section of skeletal muscle. Each polygonal structure represents a single muscle fiber. Staining for myosin ATPase reactivity at $\mathrm{pH} 4.2$ reveals grouping of both histochemical type I (darkly stained) and type II (lightly stained) fibers. Scale bar: $100 \mu \mathrm{m}$.

the lower extremities (quadriceps and tibialis anterior) showed unstable polyphasic potentials without activity at rest, a non-specific sign that did not help to determine pathology. These abnormalities were more prominent in the distal musculature. Nerve conduction studies were done to distinguish between axonal and demyelinating pathologies. They revealed mild slowing of the conduction velocity ( 35 to $45 \mathrm{~m} / \mathrm{sec}$; normal range 42 $47 \mathrm{~m} / \mathrm{sec}$ in the legs and $50-55 \mathrm{~m} / \mathrm{sec}$ in the arms) and borderline small amplitudes in the tibial, median and superficial radial nerves bilaterally. These findings do not clearly identify the problem as purely demyelinating or axonal in nature. The slight decrease in conduction velocity may reflect restricted demyelination or may alternatively reflect slowing of conduction through entrapment sites (see Discussion) or changes in nerve constitution (e.g. reduced number of large diameter, fast-conducting fibres). Reduction in the amplitude of the compound action potential is also consistent with the latter two interpretations. These electrophysiological findings must be interpreted in light of biopsy results described below.

Nerve and muscle biopsies were performed to further investigate the neuropathy. Epon sections of skeletal muscle revealed mild variation in fiber size and a few angulated fibers. There was an increase in the number of internally placed myonuclei. No evidence of inflammation was found. On cryostat sections, there were large groups of small diameter muscle fibers (Figure 1). Myosin ATPase histochemistry showed that there was a moderate degree of grouping of both type I and type II myofibers (Figure 1). Target structures were not seen. Together, these findings reflect changes that are secondary to a primary neuropathic process that has caused partial denervation and reinnervation of groups of muscle cells.

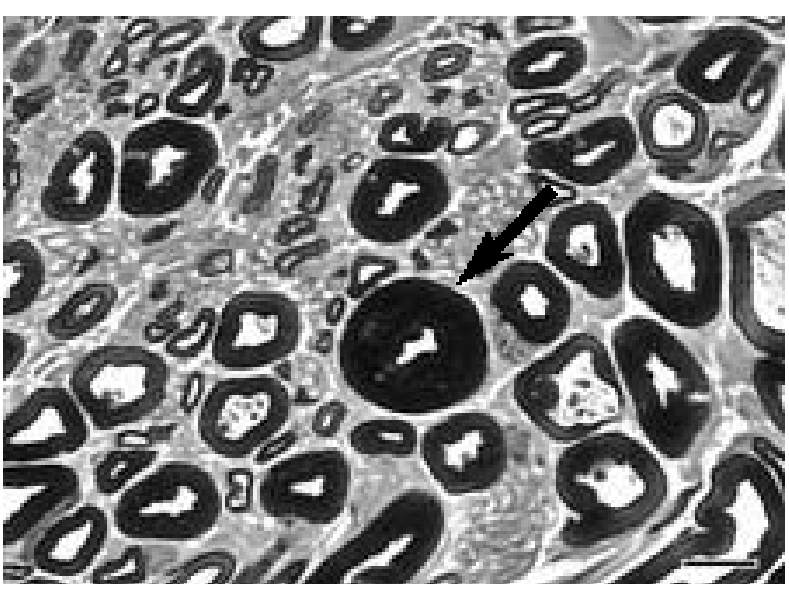

Figure 2 Cross-sections of peripheral nerve stained with the paraphenylenediamine and viewed under phase optics. This shows a relatively well populated nerve. Arrow indicates a single myelinated fiber with a markedly thickened myelin sheath. Scale bar: $10 \mu \mathrm{m}$.

Epon sections of the peripheral nerve showed a normal number of axons (Figure 2). However, there was a diminution of large caliber myelinated fibers, with an increase in the proportion of smaller diameter fibers. Several fibers had markedly thick myelin sheaths (tomaculae) in cross section. "Onion bulbs" and Wallerian degeneration were not identified.

Over 40 individual nerve fibers were teased apart and visualized by myelin staining (Figure 3 ). This analysis revealed that approximately $10 \%$ of fibers had focal sausage-shaped thickenings (tomaculae) of the myelin sheath. These thickenings were generally present near the paranodal region and sometimes more than one was observed along the same fiber. There were segmental differences in the thickness of myelin in adjacent internodes (ensheathed by different Schwann cells) as well as segmental differences in the length of the internodes, suggesting remyelination.

\section{DISCUSSION}

The patient in the case above shows evidence of both motor and sensory changes. The pattern of these changes indicate lower motor neuron degeneration, which is characterized in this patient by weakness and hyporeflexia. The longstanding history of distal weakness and the presence of pes cavus and hammer toes suggest that the neuropathy is hereditary rather than acquired. From the clinical presentation, the differential diagnosis of the peripheral neuropathy includes a form of hereditary sensory motor neuropathy (HMSN), a vasculitis, and hereditary neuropathy with liability to pressure palsies (HNPP; also known as tomaculous neuropathy).

After the initial work-up, the diagnosis of HMSN, specifically Charcot-Marie-Tooth disease type II (CMTII), was considered since nerve conductions showed 


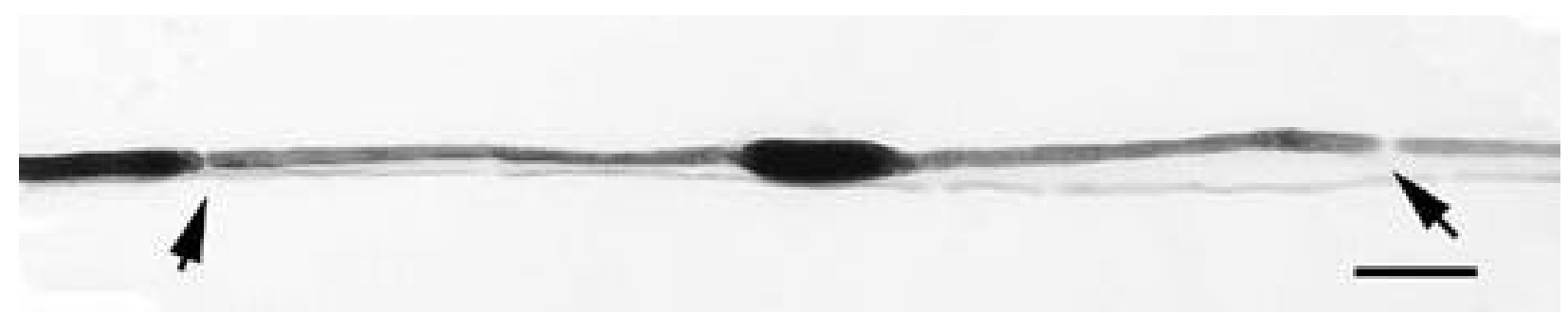

Figure 3. A small nerve bundle was fixed and stained with osmium tetroxide before individual myelinated fibers were teased out and visualized under brightfield optics. A single teased nerve fiber is shown. Arrows designate the nodes of Ranvier. The segment of myelin between the nodes is referred to as the internode and is produced by a single Schwann cell. Note the sausage-shaped enlargement of the myelin in the center of the internode. This tomaculum (Latin = sausage) corresponds to the thick myelin also noted in figure 2, and is the basis for one of the synonyms for this disease, namely tomaculous neuropathy. Scale bar: $50 \mu \mathrm{m}$.

decreased amplitudes consistent with an axonal type of neuropathy. However, the history was not consistent with CMT-II, which usually gives symmetrical neuromuscular deficits and minimal sensory symptoms. CMT-I was considered less likely since the nerve conductions were not significantly reduced in velocity as is characteristic of this disease (1). While a peripheral nerve vasculitis usually shows asymmetric and variable onset, which is consistent with this patient's presentation, the normal immunological profile and longstanding history do not support this diagnosis.

HNPP is a peripheral nerve disorder that is characterized by a susceptibility to pressure palsies, which results in both motor and sensory symptoms. In the case presented above, the asymmetric onset and progression, as well as the fluctuations in sensory symptoms are compatible with the clinical findings in HNPP. This disorder generally presents with isolated peripheral nerve dysfunction that occurs after minor trauma (such as lying awkwardly on a limb) or compression of a nerve (2). Patients may recall episodes where they feel that a limb has "gone to sleep" after a period of nerve compression. The result is a mononeuropathy in the distribution of a specific peripheral nerve. Though any nerve can be involved, commonly affected nerves include the radial nerve in the spiral groove of the humerus, the ulnar nerve at the elbow, the median nerve at the wrist and the peroneal nerve at the head of the fibula. Palsies of these nerves produce a painless, sudden onset of weakness and sensory loss over their distribution. Often, symptoms regress within a few days, however they can remain permanently. As in this case, HNPP generally first manifests itself when patients are in their 20s or 30 s. Patients may not be aware of the disorder unless the weakness and paresthesia of the mononeuropathy are associated with a specific event or injury.

Physical examination in HNPP often reveals weakness and sensory loss attributable to a specific nerve distribution. However, if the disease is advanced and many nerves are affected, findings may be generalized as in the patient described here, thereby mimicking a distal sensorimotor peripheral neuropathy such as CMT-I. The symptoms then include a decrease in distal limb reflexes, weakness and wasting of the intrinsic muscles of the hand and foot, and a decreased vibration sense.

Primary prevention is the only way to control HNPP, as there is no effective treatment for the pressure palsies. Patients can generally learn to prevent injury by avoiding pressure in susceptible nerves and thus reduce morbidity. Individual palsies can be treated with splints and physiotherapy to avoid joint contractures. Longevity is normal and quality of life is generally little affected by the disorder (3).

In HNPP, needle EMG studies are normal in muscles innervated by clinically unaffected nerves. In mild cases, the EMG shows a reduction in voluntarily activated motor unit potentials. In severe cases, signs of active denervation such as increased insertional activity or fibrillation potentials are more prominent. In this case, these findings were absent. However, significant pathology was confirmed by the presence of unstable polyphasic motor potentials, i.e. fluctuations in amplitude, duration or shape of the motor unit potential. These are nonspecific findings in both myopathic and neurogenic disturbances. The nerve conduction studies in the case above were consistent with typical HNPP findings, which usually show a widespread polyneuropathy. Motor and sensory conduction abnormalities are found not only in affected nerves, but also in those that are clinically normal. Unaffected nerves show prolongation of distal latency, mild slowing of conduction velocities, and reduced amplitude. Affected nerves show segmental slowing or block, especially at entrapment sites. There is a decrease in amplitude of the compound muscle action potential when the nerve is stimulated proximal to the block (4).

In HNPP, biopsies of clinically affected muscles typically show evidence of reinnervation, implying a low-grade denervating process affecting motor nerves 


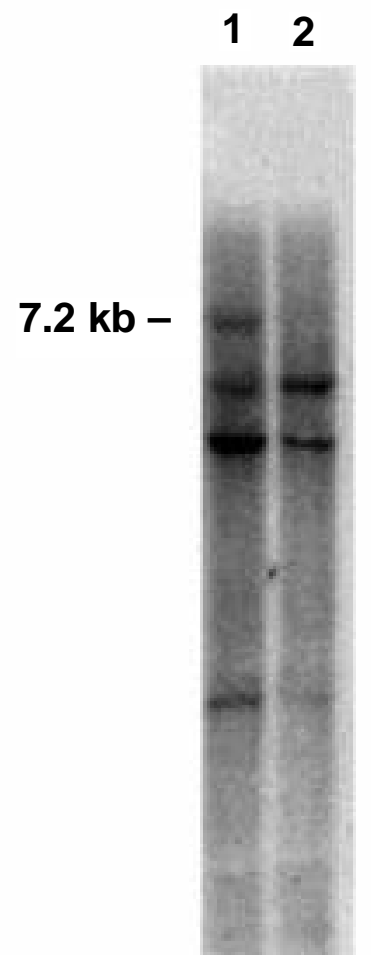

Figure 4. Genomic Southern blot from this patient (lane 1) shows recombination at the CMTIA-REP giving a novel $7.8 \mathrm{~kb}$ band characteristic for the $1.5 \mathrm{Mb}$ deletion encompassing the PMP22 gene on chromosome 17, compared to a patient exhibiting a normal PMP22 locus (lane 2).

(5). The muscle biopsy of this patient demonstrated a number of features consistent with a denervationreinnervation process: variation in muscle fiber size, large sheets of smaller diameter fibers, and fiber-type grouping. The nerve biopsy showed a shift to smaller diameter nerve fibers, which corresponds to the slowed velocities seen on nerve conduction studies. As is characteristic in HNPP, both affected and unaffected nerves of the patient showed areas of myelin thickening (tomaculae) that are particularly convincing in the teased nerve fiber preparations.

In this case, though the clinical history and physical findings were consistent with HNPP, they were relatively non-specific. The tomaculae found on microscopy strongly suggested HNPP, though they were not conclusive since tomaculae are also seen in other neuropathies such as CMT-I (6), autosomal recessive childhood neuropathy (7) and IgM monoclonal gammopathy associated with neuropathy (8). The diagnosis of HNPP was confirmed by a genomic Southern blot, which showed a novel junction fragment that is a consequence of a deletion of the PMP22 (peripheral myelin protein) gene (Figure 4).

The majority of cases of HNPP are caused by a 1.5
$\mathrm{Mb}$ deletion of chromosome 17 that encompasses the PMP22 gene. PMP22 is a hydrophobic $22 \mathrm{kDa}$ glycoprotein that is a component of peripheral nervous system myelin (9). In vitro studies $(10,11)$ suggest a role for PMP22 in the regulation of Schwann cell proliferation and apoptosis. Studies show that PMP22 may be involved in nerve growth and development, specifically in the initial spiraling of myelin during peripheral nerve growth, the determination of myelin thickness, and the maintenance of myelin and axons in the peripheral nerves (12-14). Though the role of PMP22 has been somewhat elucidated, it is unclear what the tomaculae described in nerve teases represent. Tomaculae might reflect the abnormal formation of myelin, they may be an unstable focus that is susceptible to injury or they could be the result of an abnormal response by the nervous system to injury. Nevertheless, tomaculae are a useful marker of myelin pathology in HNPP.

HNPP is allelic with CMT type IA, which has a duplication of the PMP22 gene. The classification of hereditary neuropathies is somewhat confusing, but the most useful categorization incorporates both the clinical picture and the genetics. The Charcot-Marie-Tooth syndrome refers to a group of hereditary diseases characterized by distal muscle wasting, pes cavus and sensory disturbances. The two main types, CMT type I and CMT type II, are called the "demyelinating" and "axonal" forms respectively, based predominantly on whether the electrodiagnostic studies show significant conduction slowing (decreased nerve conduction velocities) or axonopathy (decreased amplitude of compound action potentials). CMT-I is further subdivided based on whether the defect maps to the PMP22 gene on chromosome 17 (CMT-IA), or to mutations in myelin protein zero on chromosome 1 (CMT-IB). An X-linked form (CMT-X) is clinically intermediate between CMT-I and CMT-II, and is due to mutations in the gap junction protein, connexin 32. It is now possible to offer DNA-based testing for CMT-IA, CMT-IB, CMT-X, and HNPP.

This case of HNPP is unusual in that the clinical signs and symptoms are severe enough to suggest a form of CMT. Since HNPP and CMT-IA represent reciprocal DNA deletion and duplication events, respectively, it has been suggested that they should be equally prevalent in the general population (approximately 1 in 2500) (15). Clinically, HNPP is diagnosed much less commonly than CMT-IA. This could reflect the relatively benign and non-specific nature of many cases of HNPP resulting in underdiagnosis. Additionally, in more severe cases the patient could be misdiagnosed with either CMT-I or CMT-II. We feel that it is important to be aware of HNPP since it can present as a 
relatively nonspecific mono- or polyneuropathy. It is also important to recognize that the HNPP deletion is inherited in an autosomal dominant fashion.

Despite the often non-specific nature of the clinical presentation of HNPP, it is possible to make a very specific diagnosis using DNA-based testing for the HNPP deletion. This case illustrates that nerve and muscle biopsies have a role in evaluating patients who might be candidates for DNA-based testing for HNPP and other hereditary sensorimotor neuropathies.

\section{ACKNOWLEDGEMENTS}

We wish to thank the McGill Medical Informatics Project. For more neuropathology cases, please visit our website at http://sprojects.mmip.mcgill.ca/neuropath/ mainset.htm.

\section{REFERENCES}

1. Harding AE, Thomas PK. The clinical features of hereditary motor and sensory neuropathy types I and II. Brain 103: 259280; 1980.

2. Davies DM. Recurrent peripheral-nerve palsies in a family. Lancet 2: 266-268; 1954.

3. Dyck PJ, Thomas PK, Lambert R, et al. (eds). Peripheral Neuropathy, 3rd edition. Toronto: W.B. Saunders Company; 1993.

4. Behse F, Buchthal F, Carlsen F. Hereditary neuropathy with liability to pressure palsies: electrophysiological and histopathological aspects. Brain 95: 777-794; 1972.

5. Nelson JS, Parisi JE, Schichet SS (eds.). Principles and Practice of Neuropathology, 3rd Edition. St. Louis: Mosby; 1993.

6. Madrid R, Bradley WG, Davis CJF. The peroneal muscular atrophy syndrome. Journal of Neurological Sciences 32: 91-122; 1977.

7. Malandrini A, Guazzi GC, Federico A. Sensori-motor polyneuropathy in two siblings: atypical presentation of tomaculous neuropathy. Clinical Neuropathology 11: 318-322; 1992.

8. Nardelli E, Pizziguella S, Tridente G, Rizzuro N. Peripheral neuropathy associated with immunological disorders. An immunological and ultrastuctural study. Acta Neuropathologica suppl VII 258-261; 1981.

9. Snipes GJ, Suter U, Welcher AA, Shooter EM. Characterization of a novel peripheral nervous system myelin protein (PMP22/SR13). Journal of Cell Biology 117: 225-38; 1982.

10. Fabretti E, Edomi P, Brancolini C, Schneider C. Apoptotic phenotype induced by overexpression of wild-type gas3/PMP22: its relation to the demyelinating peripheral neuropathy CMT1A. Genes and Development 9: 1846-1856; 1995.

11. Zoidl G, Blass-Kampmann S, D'Urso D, et al. Retroviralmediated gene transfer of the peripheral myelin protein PMP22 in Schwann cells: modulation of cell growth. The EMBO Journal 14: 1122-1128; 1995.

12. Suter U, Moskow JJ, Welcher AA, et al. A leucine-to-proline mutation in the putative first transmembrane domain of the 22$\mathrm{kDa}$ peripheral myelin protein in the trembler-J mouse. Proceedings of the National Academy of Sciences USA 89: 4382-4386; 1992.

13. Adlkofer K, Martini R, Agussi A, et al. Hypermyelination and demyelinating peripheral neuropathy in pmp22-deficient mice. Nature Genetics 11: 274-280; 1995.

14. Magyar JP, Martini R, Ruelicke T, et al. Impaired differentiation of Schwann cells in transgenic mice with increased PMP-22 gene dosage. Journal of Neuroscience 16: 5351-5360; 1996.

15. Skre H. Genetic and clinical aspects of Charcot-Marie-Tooth's disease. Clinical Genetics 6: 98-118; 1974.

Hannah Glass is a third year medical student at McGill University. She holds a B.A. in Biology/Psychology from Columbia University, New York, New York, USA. Myriam Srour is a third year medical student at McGill University. She holds a B.Sc. in Biology from McGill University, Montreal, Quebec, Canada. Their work with the Neuropathology Department at the Montreal Neurological Institute was completed as part of the Molson Medical Informatics Project, a program designed to integrate informatics into medical education. 